\title{
Integrable Boundary Interactions
}

\author{
A.B. Zamolodchikov ${ }^{* \dagger}$ \\ Department of Physics \& Astronomy \\ Rutgers University, Piscataway NJ 08855 \\ E-mail: Sashaz@physics.rutgers.edu
}

I discuss some recent developments about integrable models of boundary interactions. I concentrate attention on the models having free massless (conformal) bulk dynamics, with the interaction solely due the nonlinear boundary conditions. I discuss relation between the boundary states of such models, infinite sets of commuting local Integrals of Motion, and the Baxter's $\mathbb{T}$ - and $\mathbb{Q}$ operators. I say few words about remarkable connection to ordinary differential equations, due to Dorey and Tateo. Although the meaning of this connection is yet to be understood, I present generalization suitable for exact solution of the so called "circular brane" model of boundary interaction, which has interesting applications in condensed matter theory.

Bethe Ansatz: 75 years later

October 19-21 2006,

Brussels, Belgium

\footnotetext{
* Speaker.

$\dagger$ This talk is based on joint works with V.Bazhanov, S.Lukyanov, E.Vitchev, and A.Tsvelick, Ref's [1-4]. Parts of unpublished work with S.Lukyanov [5] are also included. It is my pleasure to express gratitude to them for collaboration.
} 
Integrable quantum field theories with boundary interactions are among many developments made possible thanks to Bethe's original discovery. Lately, the boundary interactions attract much attention, for variety of reasons. For one, such models have many applications in many-body theory, including the Kondo problem and dissipative quantum mechanics. Another area is the string theory, where understanding of boundary interactions is expected to give insight into the configuration space of the open string field theory. In this talk I discuss some recent developments about integrable boundary interactions. I will concentrate attention on the models with free massless bulk, with the interaction emerging only from the nonlinear boundary conditions ${ }^{1}$. There is a remarkable relation between the boundary states of such models and the Baxter's $\mathbb{T}$ - and $\mathbb{Q}$ - operators, which allows one to study integrable structures in quantum field theory, in relatively simple settings. Besides, some models of this kind have direct applications in condensed matter physics. The talk is based mostly on the papers [1, 2, 3, \#, where more details as well as references to earlier works, can be found.

Generally, when constructing an integrable boundary interaction one starts with a bulk field theory which is integrable. Thus we assume that the bulk theory has infinite set of local currents $J_{n}^{\mu}=\left(J_{n}^{x}, J_{n}^{y}\right)$, each satisfying the continuity equation

$$
\partial_{\mu} J_{n}^{\mu} \equiv \partial_{x} J_{n}^{x}+\partial_{y} J_{n}^{y}=0
$$

and that the associated charges $Q_{n}=\int J_{n}^{y}(x, y) d x$ commute

$$
\left[Q_{n}, Q_{m}\right]=0 .
$$

Here I discuss in terms of the Euclidean version of the QFT, and $(x, y)$ are Cartesian coordinates of $2 D$ space, with $y=-i t$ interpreted as the Euclidean time.

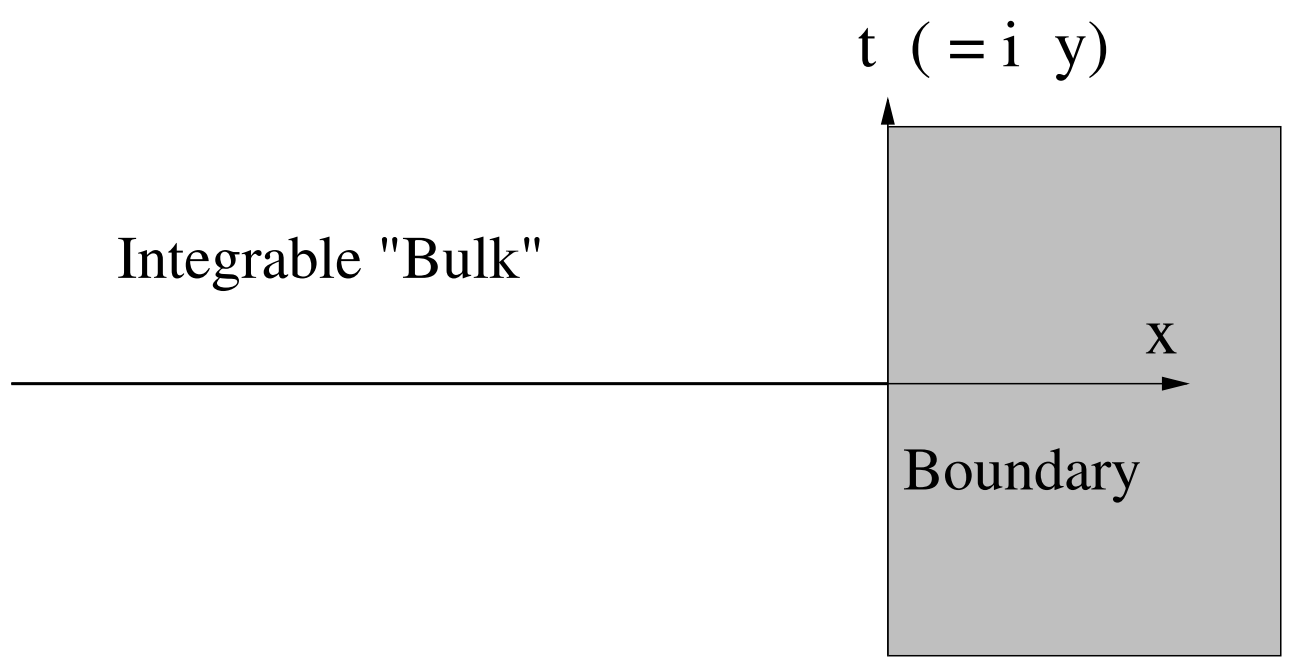

Next, we assume that the boundary is present at $x=0$, as shown in Fig.1. In order to preserve the integrability, one needs to choose special boundary conditions at $x=0$, such that appropriately

\footnotetext{
${ }^{1}$ In more general context, integrable models with boundary of course have long history beginning with the pioneering works Cherednik and Sklyanin, which I do not attempt to review. Furthermore, the citations I give are not meant to be complete or systematic. I mention only works directly relevant for the discussion.
} 
modified charges $Q_{n}$ still conserve. One easily checks that it is sufficient to have

$$
\left.J_{n}^{x}(x, y)\right|_{x=0}=\frac{d}{d y} \Theta_{n}(y),
$$

where $\Theta_{n}(y)$ are some local boundary fields. Then the charges

$$
Q_{n}=\int_{-\infty}^{0} J_{n}^{y}(x, y) d x+\Theta_{n}(y)
$$

are independent on the "time" $y$.

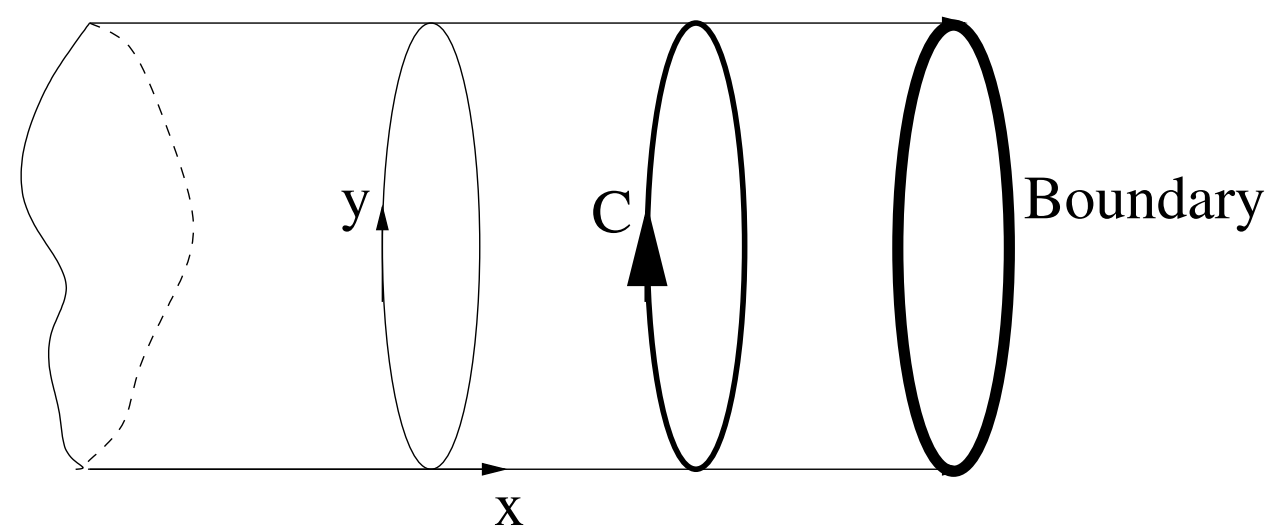

For reasons which will become clear later, we will assume that the coordinate $y$ is compactified on a circle with a circumference $2 \pi R$, as shown in Fig.2. This of course is the same as taking the field theory in a thermal equilibrium state, with the temperature $T=(2 \pi R)^{-1}$.

Alternative interpretation is achieved by interchanging the roles of the coordinates $x$ and $y$ as the space and time. Taking now $x$ as the Euclidean time, we have have a system in which the space is a circle, with no boundary. Operator quantization of such system leads to the space of states $\mathscr{H}_{\text {closed }}$ which knows nothing about the boundary at $x=0$. The boundary at $x=0$ appears now as a sort of initial condition, i.e. it generates certain state

$$
|B\rangle \in \mathscr{H}_{\text {closed }}
$$

so that expectation values are represented as the matrix elements

$$
\langle\cdots\rangle=\frac{\langle 0|\cdots| B\rangle}{\langle 0 \mid B\rangle}
$$

where $|0\rangle$ is the ground state in this Hamiltonian picture.

The theory still have conserved (i.e. $x$-independent) charges associated with the currents (1),

$$
\mathbb{H}_{n}=\int_{0}^{2 \pi R} J^{x}(x, y) d y .
$$

In quantum theory this integrals are operators acting in $\mathscr{H}_{\text {closed }}$, and by our assumption they form commutative set,

$$
\mathbb{H}_{n}: \mathscr{H}_{\text {closed }} \rightarrow \mathscr{H}_{\text {closed }}, \quad\left[\mathbb{H}_{n}, \mathbb{H}_{m}\right]=0
$$


Note that now the charges (4) do not involve terms related to the boundary conditions at $x=0$. Therefore their conservation depends only on the continuity equations (11), but does not require the conditions (3). What are the implications of the Eqs.(3) in this picture? Since the integrals (7) do not depend on $x$, we can send $x$ to 0 , where the equations (3) apply. As the result the integrands in (7) become total derivatives, and the integrals vanish. That is, the local conditions (3) are implemented as the constraints on the boundary state,

$$
\mathbb{H}_{n}|B\rangle=0
$$

Thus, constructing integrable boundary interaction involves two steps: (a) Understanding the structure of the space $\mathscr{H}_{\text {closed }}$ (in other words, finding solution of the integrable theory in the bulk), and (b) Identifying the boundary state (5), subject to the integrability constraint $(9)^{2}$. In this discussion I will concentrate attention of special case where the first step is trivial. Namely, I will consider theories whose bulk dynamics is that of free massless bosons. generally, such theory involves $D$ scalar fields $\mathbf{X}=\left(X^{1}, x^{2}, \ldots, X^{D}\right)$, with the bulk action

$$
\mathscr{A}_{\text {bulk }}=\frac{1}{4 \pi} \int_{-\infty}^{0} d x \int_{0}^{2 \pi R} d y\left(\partial_{a} \mathbf{X}\right)^{2}
$$

where I assumed the $2 D$ geometry as in Fig.2. Then, the interaction can be generated by adding non-linear boundary conditions at the boundary $x=0$. Generally, it can be done in two ways:

I. By adding a nonlinear boundary term to the action,

$$
\mathscr{A}=\mathscr{A}_{\text {bulk }}+\int_{0}^{2 \pi R} V\left(\mathbf{X}_{B}(y), \Xi(y)\right) d y,
$$

which can depend only on the boundary values

$$
\left.\mathbf{X}_{B}(y) \equiv \mathbf{X}(x, y)\right|_{x=0}
$$

of the fields; it can also involve additional boundary degrees of freedom $\Xi(y)$ (like the impurity spin in the Kondo problem) which have no bulk counterparts.

II. By imposing nonlinear constraints on the boundary values $\mathbf{X}_{B}$ :

$$
\mathbf{X}_{B} \in \Sigma \subset \mathbb{R}^{D} \quad\left({ }^{\prime \prime} \text { Brane }^{\prime \prime}\right)
$$

where $\Sigma$ is a sub-manifold embedded in the "target space" $\mathbb{R}^{D}$.

Of course, combinations of I and II are possible. There are two reasons to be interested in this class of models. One is the technical advantage already mentioned above: the solution of the bulk theory, and the space of states $\mathscr{H}_{\text {closed }}$ are understood upfront. Besides, these models have interesting physical interpretations, as the models of quantum Brownian motion: The boundary values $\mathbf{X}_{B}(y)$ are interpreted as the coordinates of a Brownian particle (with $y=-i t$ being imaginary

\footnotetext{
${ }^{2}$ Of course, boundary state must be generated by a local boundary condition. Therefore true boundary states constitute very special subset (not a subspace!) in $\mathscr{H}_{\text {closed }}$. Unfortunately, in general no useful abstract characterization of the locality condition (would be generalization of the annulus bootstrap of the boundary CFT) is yet available.
} 
time), while the bulk part of $\mathbf{X}$ plays the role of Caldeira-Leggett quantum thermostat, generating friction and noise.

In any case, we would like to identify boundary interactions which preserve integrability, i.e. fulfill the conditions (9), with some $\mathbb{H}_{n}$. The free bulk theory certainly has an infinite set of commuting local integrals of the form ( ( $)$. In fact, there usually is more then one such commuting set. Let us recall general structure of the local currents in the free theory (10). It is useful to discuss it terms of the complex coordinates

$$
z=y+i x, \quad \bar{z}=y-i x
$$

The bulk dynamics is such that the field splits into the left- and right- moving parts,

$$
\mathbf{X}(z, \bar{z})=\mathbf{X}_{L}(z)+\mathbf{X}_{R}(\bar{z})
$$

implying that any local polynomial which involves only $z$-derivatives

$$
P_{s+1}\left(\partial_{z} \mathbf{X}, \partial_{z}^{2} \mathbf{X}, \ldots, \text { higher derivatives }\right)
$$

is a holomorphic field

$$
\partial_{\bar{z}} P_{s+1}=0 \quad \Rightarrow \quad P_{s+1}=P_{s+1}(z) .
$$

Below I always assume that the polynomials are homogeneous in the number of the derivatives, which is indicated as the spin $s+1$ of the field $P_{s+1}$. The equation (17) is a special case of (11), and the associated integral of the form (7)

$$
\mathbb{I}_{s}=\int_{0}^{2 \pi R} P_{s+1}\left(\partial_{z} \mathbf{X}, \partial_{z}^{2} \mathbf{X}, \ldots\right) d z
$$

conserves. Of course, there "right-moving" counterparts to these integrals of motion, $\overline{\mathbb{I}}_{s}=\int_{0}^{2 \pi R} \bar{P}_{s+1} d \bar{z}$, where $\bar{P}_{s+1}$ are the same polynomials (16) with the $z$-derivatives replaced by the $\bar{z}$-derivatives. By construction, the right integrals commute with the left ones, $\left[\mathbb{I}_{s}, \overline{\mathbb{I}}_{s^{\prime}}\right]=0$. But the full set of, say, the left integrals of the form (18), with all independent $P_{s+1}$, form non-commutative algebra. What we need is a commutative subalgebra(s) thereof.

Complete classification of such commutative subalgebras is an interesting mathematical problem, which remains largely open. Some explicit constructions are known. The best known is the so called $K d V$ series of commuting IM. It is one-parameter family realized in the case $D=1$ by infinite set of currents $P_{s+1}^{K d V}$ with $s=1,3,5,7, \ldots$. Explicitly

$$
\begin{aligned}
P_{2}^{K d V} & =(\partial X)^{2} \\
P_{4}^{K d V} & =\left(\partial_{z} X\right)^{4}+\left(1-\alpha_{0}\right)\left(\partial_{z}^{2} X\right)^{2}+\text { derivatives }, \\
\ldots & \quad \text { Energy }- \text { Momentum tensor }) \\
P_{2 k}^{K d V} & =\left(\partial_{z} X \partial_{z} X\right)^{2 k}+\frac{k(k-1)(2 k-1)\left(2 k-3-\alpha_{0}\right)}{6}\left(\partial_{z}^{2} X\right)^{2}\left(\partial_{z} X \partial_{z} X\right)^{2 k-4}+\cdots
\end{aligned}
$$


where $\alpha_{0}$ ia the parameter ${ }^{3}$. Next, one can prove existence, and in many cases give explicit construction, of several commutative subalgebras associated with $\mathrm{W}$-algebras and their reductions. Is there anything beyond these "regular" series? As far as I know only sporadic results obtained by brute-force calculations are available, but they suggest that the problem is far from being exhausted. I will mention here one example only.

Let us restrict attention to currents invariant with respect to orthogonal rotations of the target space $\mathbb{R}^{D}$. That means the polynomials $P_{s+1}$ may only involve the $O(D)$-invariant terms, i.e.

$$
\begin{array}{ll}
P_{2}^{O(D)} & =\left(\partial_{z} \mathbf{X} \partial_{z} \mathbf{X}\right) \quad(\text { Energy }- \text { Momentum tensor }) \\
P_{4}^{O(D)} & =\left(\partial_{z} \mathbf{X} \partial_{z} \mathbf{X}\right)^{2}+b_{3}\left(\partial_{z}^{2} \mathbf{X} \partial_{z}^{2} \mathbf{X}\right) \\
P_{6}^{O(D)} & =\left(\partial_{z} \mathbf{X} \partial_{z} \mathbf{X}\right)^{3}+b_{5}\left(\partial_{z}^{2} \mathbf{X} \partial_{z}^{2} \mathbf{X}\right)\left(\partial_{z} \mathbf{X} \partial_{z} \mathbf{X}\right)+c_{5}\left(\partial_{z}^{2} \mathbf{X} \partial_{z} \mathbf{X}\right)^{2}+d_{5}\left(\partial_{z}^{3} \mathbf{X} \partial_{z}^{3} \mathbf{X}\right)
\end{array}
$$

etc

Our goal is to adjust the coefficients $b_{3}, b_{5}, c_{5}$, etc, in such a way that the integrals (18) commute. This condition turns out to be surprisingly rigid. While the conditions $\left[\mathbb{I}_{s}, \mathbb{I}_{1}\right]=0$ are satisfied under any choice of these coefficients in a trivial way, the first nontrivial equation

$$
\left[\mathbb{I}_{3}, \mathbb{I}_{5}\right]=0
$$

fixes the coefficients in $P_{4}$ and $P_{6}$ above essentially uniquely. More precisely, there are three solutions of these equations. Two of them are not unexpected: one is the KdV series associated with the Virasoro algebra which was already mentioned above, and another is the the "free-field" series where all the currents $P_{s+1}$ are quadratic in $\mathbf{X}$. But the third solution is new:

$$
\begin{aligned}
& b_{3}=\frac{D+2}{3}, \quad b_{5}=\frac{3(D+4)}{5}, \\
& c_{5}=\frac{7(D+4)}{5}, \quad d_{5}=\frac{(D+4)(36 D+59)}{600} .
\end{aligned}
$$

Once these coefficients are fixed, the equations $\left[\mathbb{I}_{3}, \mathbb{I}_{s}\right]=0$ determine the higher currents

$$
P_{2 k}=\left(\partial_{z} \mathbf{X} \partial_{z} \mathbf{X}\right)^{k}+b_{2 k-1}\left(\partial_{z}^{2} \mathbf{X} \partial_{z}^{2} \mathbf{X}\right)\left(\partial_{z} \mathbf{X} \partial_{z} \mathbf{X}\right)^{k-2}+\cdots
$$

uniquely,

$$
b_{2 k-1}=\frac{k(k-1)(2 k+D-2)}{2(2 k-1)}, \quad c_{2 k-1}=\frac{k(k-1)(k-2)(2 k+1)(2 k+D-2)}{6(2 k-1)} .
$$

I will refer to this series of commuting integrals of motion as the $O(D)$ series. Below I describe integrable boundary condition associated with this series. At this moment my point is bringing

\footnotetext{
${ }^{3}$ These currents can be written, up to total derivatives, as the composites of the "modified EM tensor" $T=$ $-(\partial X)^{2}+i \alpha_{0} \partial^{2} X$, which generates the Virasoro algebra with $c=1-6 \alpha_{0}^{2}$. Thus,

$$
P_{2}^{K d V}=-T+\text { derivatives }, \quad P_{4}^{K d V}=T^{2}+\text { derivatives }, \quad P_{6}^{K d V}=T^{3}-\frac{c+2}{12}(\partial T)^{2}, \quad \text { etc }
$$
}


attention to this interesting and mostly open problem - classification of commutative subalgebras in the algebra of local integrals (18).

Once the set $\left\{\mathbb{I}_{s}\right\}$ of commuting integrals is selected, we turn attention back to the boundary state which satisfies the Eqs.(9). How the operators $\mathbf{H}_{s}$ is (9) relate to the integrals (18)? As will become clear in a moment, it is natural, indeed necessary, to take

$$
\mathbb{H}_{s}=\mathbb{I}_{s}-\overline{\mathbb{I}}_{s} .
$$

Note that the equations

$$
\left(\mathbb{I}_{s}-\overline{\mathbb{I}}_{s}\right)|B\rangle=0
$$

are somewhat similar to the conditions of conformal invariance

$$
\left(\mathbb{L}_{n}-\overline{\mathbb{L}}_{-n}\right)\left|B_{\text {conf }}\right\rangle=0,
$$

well known in boundary CFT. And as in the CFT, they also can be written as certain commutativity conditions. Indeed, because of the left- right separation (15), the space $\mathscr{H}_{\text {closed }}$ admits holomorphic factorization

$$
\mathscr{H}_{\text {closed }}=\int_{\mathbf{P}} \mathscr{F}_{\mathbf{P}} \otimes \overline{\mathscr{F}} \mathbf{P}
$$

well-known general feature of CFT, where in this case $\mathscr{F}$ p and $\overline{\mathscr{F}} \mathbf{P}$ are the Fock spaces associated with the left- and right- movers in (15), with the zero-mode momentum $\mathbf{P}$. The space (28) is a subspace of a direct product of two isomorphic spaces. There is a natural correspondence between states in such space and endomorphisms of the Fock spaces:

$$
\mathscr{F} \otimes \mathscr{F} \quad \leftrightarrow \quad \operatorname{End}(\mathscr{F}) .
$$

Let $\mathbb{B}$ be operator associated in this manner with the boundary state:

$$
|B\rangle \in \mathscr{F} \otimes \mathscr{F} \quad \mathbb{B} \in \operatorname{End}(\mathscr{F}) .
$$

I will refer to $\mathbf{B}$ as the boundary state operator. Note that this correspondence owes its existence to the holomorphic factorization, i.e. ultimately to the conformal character of the bulk theory; the boundary state operator acts in the chiral component $\mathscr{F}=\int_{\mathbf{P}} \mathscr{F}_{\mathbf{P}}$, not in the full space (28). In fact, they act in each component $\mathscr{F} \mathbf{P}$ separately,

$$
\mathbb{I}_{S}: \quad \mathscr{F}_{\mathbf{P}} \rightarrow \mathscr{F}_{\mathbf{P}} .
$$

It is easy to check that the equation (26) is equivalent to the commutativity condition

$$
\left[\mathbb{I}_{s}, \mathbb{B}\right]=0 \quad \text { for all } \mathbb{I}_{s} .
$$

It is instructive to compare it with the conformal case, where the equation (27) writes as

$$
\left[\mathbb{L}_{n}, \mathbb{B}_{\text {conf }}\right]=0 \quad \text { for all } \mathbb{L}_{n}
$$


Here $\mathbb{L}_{n}$ is the full set of the Virasoro algebra generators, and (33) leads to a well-known decomposition of a conformal boundary state into the Ishibashi states,

$$
\mathbb{B}_{\mathrm{conf}}=\sum_{r} b_{r} \mathbb{P}_{r}
$$

where $\mathbb{P}_{r}$ are projectors on the irreducible representations of the Virasoro algebra, and the coefficients are determined from the conditions of locality (in practice, from the Cardy's annulus modular bootstrap equations).

Now, in the Eq.(32) $\left\{\mathbb{I}_{s}\right\}$ is a commutative algebra. Let us assume that this set of operators is "resolving" one, in the sense that all simultaneous eigenvalues of $\left\{\mathbb{I}_{s}\right\}$ are one-dimensional. This is likely the case for all known sets of commuting integrals, it can be verified for few lowest levels by direct calculations. Then it follows

$$
\mathbb{B}=\sum_{a} B_{a} \mathbb{P}_{a}
$$

where now $\mathbb{P}_{a}$ are projectors on the simultaneous eigenspaces of all the integrals $\left\{\mathbb{I}_{s}\right\}$.

Unlike CFT, here we are interested in more general boundary conditions, which are integrable but not conformal. Since scale invariance is not assumed, such boundary condition should involve dimensional parameter, i.e. some characteristic energy $\lambda$. The boundary state $|B\rangle$, or equivalently the operator $\mathbb{B}$, depends on this parameter,

$$
\mathbb{B}=\mathbb{B}(\lambda) .
$$

On the other hand, the integrals $\mathbb{I}_{s}$ do not involve any scale parameter at all - recall that the bulk theory (10) has scale invariance as the part of its conformal symmetry. Hence the eigenspaces of $\left\{\mathbf{I}_{s}\right\}$ are independent of $\lambda$, and the scale can enter the equation (35) only through the coefficients $B_{a}$,

$$
\mathbb{B}(\lambda)=\sum_{a} B_{a}(\lambda) \mathbb{P}_{a}
$$

Immediate consequence is that the operators $\mathbb{B}(\lambda)$ commute at different scales,

$$
\left[\mathbb{B}(\lambda), \mathbb{B}\left(\lambda^{\prime}\right)\right]=0 .
$$

This of course is reminiscent of the Baxter's commuting families of Transfer-matrices. Since the operator $\mathbb{B}$ acts in the space $\mathscr{F}=\int_{\mathbf{P}} \mathscr{F} \mathbf{P}$, which is the chiral space of the Conformal Field Theory, one may expect relation to the Baxter's operators of the CFT. In fact, direct relation can be established in the simplest cases. For instance, the Baxter's $\mathbb{T}$ and $\mathbb{Q}$ operators associated with the $U_{q}(s l(2)) R$-matrix are explicitly constructed in the Fock space with $D=1$, and they are manifestly related to the operators $\mathbb{B}$ of the boundary sine-Gordon model. Thus, the $\mathbb{T}$-operators we have

$$
\mathbb{T}(\lambda)=\operatorname{tr}\left[e^{i \pi \beta P H} \mathscr{P} \exp \left\{\lambda \int_{0}^{2 \pi R}\left[E e^{-2 i \beta X_{L}(z)}+F e^{2 i \beta X_{L}(z)}\right] d z\right\}\right]
$$

where $E, F, H$ are generators of the $U_{q}(\operatorname{sl}(2))$ algebra with $q=e^{i \pi \beta^{2}}$,

$$
[H, E]=2 E, \quad[E, F]=-2 F, \quad[E, F]=\frac{\sinh \left(\pi \beta^{2} H\right)}{\sinh \left(\pi \beta^{2}\right)} .
$$


and the trace is taken over one of its finite-dimensional representation. Here $X_{L}(z)$ is the left-moving chiral part of the field $X$ in (15), and $P$ is its zero-mode momentum operator. The operator(s) $\mathbb{Q}(\lambda)$ admit similar representation, with $E, F, H$ replaced by generators of the so-called $q$-oscillator algebra [?]. Note that since the free-field exponentials are dimensional, the spectral parameter $\lambda$ is effectively dimensional as well, $\lambda \sim[\text { length }]^{\beta^{2}-1}$. The expression (39) explicitly have the form of the boundary state operator for the boundary interaction involving the boundary "spin" $(E, F, H)$ (it can be regarded as the anisotropic generalization of the Kondo interaction). The $\mathbb{Q}$ operator can be related to the boundary state of the boundary sine-Gordon model. Asymptotic expansions of the above Baxter's operators around the point $\lambda=\infty$ generate the local Integrals of Motion of the KdV series; for instance

$$
\log \mathbb{T}_{1 / 2}(\lambda)=C_{0} R \lambda^{\frac{1}{1-\beta^{2}}}+\sum_{k=1}^{\infty} C_{k} \mathbb{I}_{2 k-1} \lambda^{\frac{1-2 k}{1-\beta^{2}}},
$$

where the subscript $1 / 2$ in $\mathbb{T}_{1 / 2}$ indicates that the trace in (39) is taken over the 2-dimensional representation. The parameter $\alpha_{0}$ in (19) is related to $\beta$ in the standard manner

$$
\alpha_{0}=\beta-1 / \beta
$$

Connection to the Baxter's operators opens way to another remarkable tool of integrable models. It was discovered by Doorey and Tateo [6] (see also [7]that the vacuum-vacuum matrix elements of the above $U_{q}(s l(2))$ Baxter's operators are related to certain monodromy coefficients of the ordinary differential equation

$$
\left[-\frac{d^{2}}{d \eta^{2}}+\frac{l(l+1)}{\eta^{2}}+\eta^{2 \alpha}\right] \Psi(\eta)=E \Psi(\eta)
$$

where the parameters are related as

$$
2 l+1=P / \beta, \quad \alpha=1-1 / \beta^{2}, \quad \text { and } \quad E=\left(2 / \beta^{2}\right)^{2\left(1-\beta^{2}\right)} \Gamma^{2}\left(1-\beta^{2}\right) \lambda^{2} .
$$

The relation is quite remarkable - it allows to reduce calculation of certain characteristics of integrable field theory to finite-dimensional analysis (differential equations). It was established on formal level, by comparing the functional equations on the both sides of the correspondence. Unfortunately, deeper root of this relation still escaped my understanding. For instance, it would be important to find interpretation of the "coordinate" $\eta$, as well as the "wave function" $\Psi(\eta)$, in terms of the boundary quantum field theory. To my mind, this is one of the most compelling open problems in this field. Furthermore, similar relation seems to exist in many integrable models of boundary interaction, so that the solutions of such models can be incorporated in certain ordinary differential operators. Below I write down what seems to be the differential equation associated with the so-called circular brane model.

Let us go back to the $O(D)$ series of IM, the Eq.(20). What integrable boundary condition is invariant with respect to this series? Let us assume that the boundary interaction is of the "brane" type, i.e. is due to the boundary constraint (13). (The assumption is be supported by the subsequent analysis.) In view of the $O(D)$ symmetry the sub-manifold $\Sigma$ can not be anything but a sphere $\mathbb{S}^{D-1} \subset \mathbb{R}^{D}$. The boundary constraint takes the explicit form

$$
\mathbf{X}_{B}^{2}=\rho_{0}^{2} \equiv 1 / g_{0}
$$


where I introduce more convenient parameter $g_{0}$ instead of the radius of the sphere $\rho_{0}$. We refer to this boundary interaction as the "spherical brane model". It is possible to argue that the model is integrable. One has to show that there is an infinite number of local IM which satisfy the condition (9). In practice, it suffices to construct just one nontrivial (going beyond the energy-momentum) local current satisfying the equation (3). It is possible to do that explicitly for the current of the spin $s=4$, in which case we look for $J^{x}$ in the form

$$
J_{4}^{x}=P_{4}-\bar{P}_{4}, \quad P_{4}=\left(\partial_{z} \mathbf{X} \partial_{z} \mathbf{X}\right)^{2}+b_{3}\left(\partial_{z}^{2} \mathbf{X} \partial_{z}^{2} \mathbf{X}\right),
$$

with yet undetermined coefficient $b_{3}$. By enumerating all possible boundary operators which by virtue of dimension and symmetries can appear in the r.h.s. of (ß), one can verify that there is only one, modulo the equations of motion, non-derivative term of this kind, and hence the equation (B) can always be satisfied by suitable choice of $b_{3}{ }^{4}$. The fact that the $O(D)$ series (20) is the right choice of IM associated with this boundary conditions can be confirmed by explicit calculations within the $1 / D$ expansion.

The case $D=2$ goes by the name of "circular brane model". It is of special interest because of its applications in condensed matter theory. It relates to the so-called Ambegaokar-EckernSchön (AES) model which is the model of dissipative quantum mechanics commonly adopted in describing the Coulomb charging of a quantum dot, in the limit of large conduction. By integrating out the bulk part of the field $\mathbf{X}=(X, Y)$ in the functional integral with the Gaussian bulk action (10) one reduces the circular brane model to the non-local action

$$
\mathscr{A}_{\mathrm{diss}}=-H \int_{0}^{2 \pi R} \cos \varphi(y) d y+\frac{1}{8 \pi^{2} R^{2} g_{0}} \int_{0}^{2 \pi R} d y \int_{0}^{2 \pi R} d y^{\prime} \frac{\sin ^{2}\left(\frac{\varphi(y)-\varphi\left(y^{\prime}\right)}{2}\right)}{\sin ^{2}\left(\frac{y-y^{\prime}}{2 R}\right)},
$$

where $\varphi(y)$ is the angle associated with the boundary field $\mathbf{X}_{B}(y)=\left(X_{B}(y), Y_{B}(y)\right)$,

$$
X_{B}(y)=\frac{\cos \varphi(y)}{\sqrt{g_{0}}}, \quad Y_{B}(y)=\frac{\sin \varphi(y)}{\sqrt{g_{0}}},
$$

and $H$ is proportional to the zero-mode momentum $\mathbf{P}$,

$$
H=\frac{i|P|}{2 \pi R \sqrt{g_{0}}} .
$$

The Eq. (47) is exactly the dissipative action of AES ${ }^{5}$, with $g_{0}$ interpreted as the dimensionless conductance of the tunneling contact, and of course $2 \pi R=1 / T$.

\footnotetext{
${ }^{4}$ In this analysis the PT symmetry of the theory is implicit. Therefore it applies only when the topological angle $\theta$ is 0 or $\pi$. The topological angle is defined with respect to the winding number $W$, the number of times the boundary value $\mathbf{X}_{B}(y)$ winds over the circle (45) when $y$ goes around the boundary circle in Fig.2. Therefore this "counting argument" is valid only at $\theta=0, \pi$. It is not known whether the circular brane integrable at generic $\theta$ (see however [8]).

${ }^{5}$ More precisely, (43) reproduces the dissipative part of the AES action. In general, AES action involves also the term associated with the capacitive energy,

$$
\mathscr{A}_{\mathrm{AES}}=\mathscr{A}_{\mathrm{diss}}+\frac{1}{4 E_{C}} \int_{0}^{2 \pi R}\left(\varphi_{y}\right)^{2} d y, \quad E_{C}=\frac{e^{2}}{2 C},
$$

it reduces to 47 in the limit of large conductance, $g_{0} \rightarrow 0$. At small $g_{0}$ the capacitive term provides explicit UV cutoff, with the cutoff energy $\Lambda=E_{C} / g_{0}$.
} 
The solution of the circular brane model can be found by identifying the associated differential equation analogous to the Eq.(43), which turns out to have the form

$$
\left[-\frac{d^{2}}{d w^{2}}+\kappa^{2} e^{e^{w}}+h^{2} e^{w}\right] \Psi(w)=0 .
$$

Here $h=\pi \sqrt{g_{0}} R H$, and $\kappa=E_{*} R$, with $E_{*}$ being the physical energy scale of the model. The latter is related to the UV cutoff $\Lambda$ by the "RG flow equation" of the model, i.e.

$$
E_{*}=\frac{\Lambda}{g_{0}} e^{-\frac{1}{2 g_{0}}}
$$

Once solutions of the differential equation (50) are found, the partition function of the circular brane model is obtained in closed form

$$
Z\left(E_{*} R, H\right)=\sqrt{\frac{\pi}{\kappa}} \frac{\left\{2 e^{\gamma_{E}-2} \kappa^{2}\right\}^{\kappa}}{\Gamma(1+2 \kappa)}\left[\Psi_{+}, \Psi_{-}\right]
$$

where the last factor is the Wronskian $\Psi_{+}(w) \Psi_{-}^{\prime}(w)-\Psi_{+}^{\prime}(w) \Psi_{-}(w)$ of two suitably normalized solutions of (50), one $\left(\Psi_{+}\right)$decaying at $w \rightarrow+\infty$, and another $\left(\Psi_{-}\right)$decaying at $w \rightarrow-\infty{ }^{6}$.

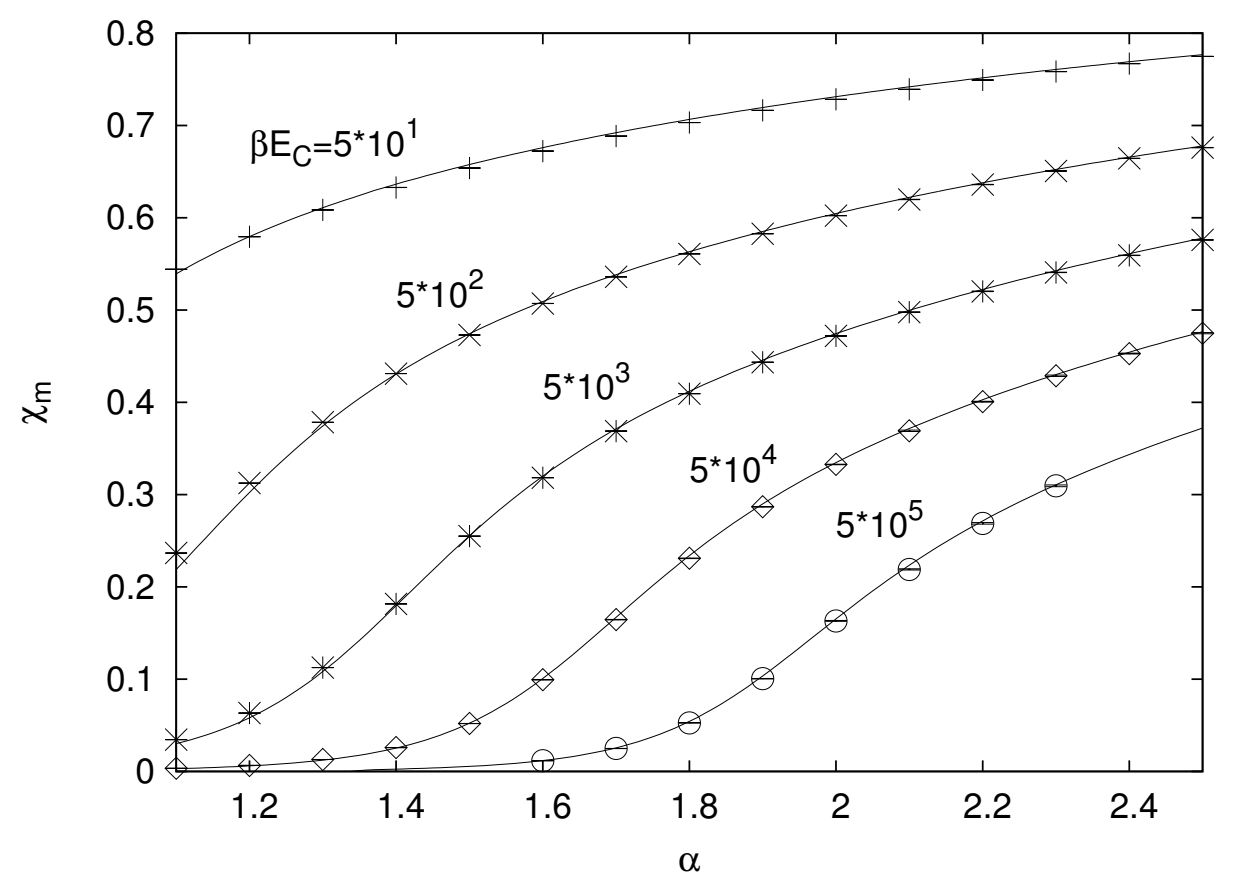

${ }^{6}$ The normalization is such that $\Psi_{+}(w) \rightarrow \exp \left(-e^{w} / 4-\kappa E i\left(e^{w} / 2\right)\right)$ as $w \rightarrow+\infty$, and $\Psi_{-} \rightarrow e^{\kappa w}$ as $w \rightarrow-\infty$. 
Comparison of the exact result with recent Monte Carlo data on the AES model is shown in Fig.3. The plot (borrowed, with permission, from Ref.[0]) shows the "magnetic susceptibility" $\chi_{m}=\frac{\partial^{2}}{\partial H^{2}} \log Z$, obtained from Eq. (52), vs the MC results, at different values of the parameter $\alpha=\left(2 \pi^{2} g_{0}\right)^{-1}$.

Let me mention two other integrable "brane" models, together with the associated differential equations.

One is the "paperclip model", generalizing the circular brane model. The constraint (45) is replaced by

$$
r \cosh \left(\frac{X_{B}}{\sqrt{n}}\right)-\cos \left(\frac{Y_{B}}{\sqrt{n+2}}\right)=0, \quad\left|Y_{B}\right| \leq \pi \sqrt{n+2} .
$$

Here $r$ and $n$ are parameters. The boundary constraint is subject to the RG flow, so that the parameter $r$ is not a constant but flows with the energy scale $E$,

$$
(n+1) r^{n}\left(1-r^{2}\right)=E_{*} / E
$$

where $E_{*}$ is the characteristic energy scale (the integration constant of the RG equation). The Eq. (53) defines the "paperclip curve" shown in Fig.4.

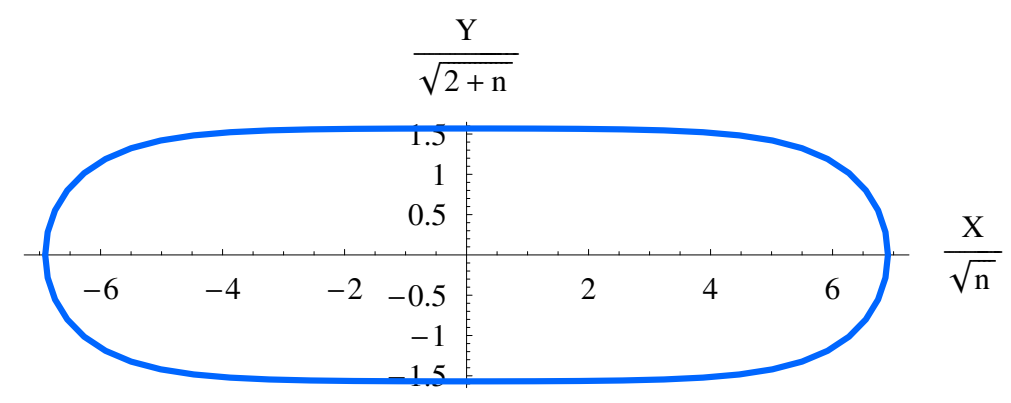

The associated differential equation is identified in Ref.[3],

$$
\left[-\frac{d^{2}}{d w^{2}}+\frac{A^{2} e^{w}}{1+e^{w}}+\frac{B^{2} e^{w}}{\left(1+e^{w}\right)^{2}}+\left(E_{*} R\right)^{2}\left(1+e^{w}\right)^{n}\right] \Psi(w)=0
$$

Here $A=-n P^{2}$ and $B=1 / 4-(n+2) Q^{2}$, where $P, Q$ are the components of the zero mode momentum, $\mathbf{P}=(P, Q)$. The circular brane model (and the differential equation (50) ) is recovered in the limit $n \rightarrow \infty$.

The "pillow" brane model [5] has three-dimensional target space, $\mathbf{X}=(X, Y, Z) \in \mathbb{R}^{3}$. The boundary constraint has the form

$$
\sqrt{\left(1+\rho_{1}\right)\left(1+\rho_{2}\right)} \cos \frac{Z_{B}}{\sqrt{n+2}}=\sqrt{\rho_{1} \rho_{2}} \cosh \frac{X_{B}}{\sqrt{v n}}+\cosh \frac{Y_{B}}{\sqrt{(1-v) n}},
$$

where $n$ and $v$ are parameters, and $\rho_{1}$ and $\rho_{2}$ are two roots of the algebraic equation

$$
\rho^{n v}(1+\rho)^{-(n+2)}\left(n(1-v)(2+n(1-v)) \rho^{2}+2 n^{2} v(1-v) \rho+n v(2+n v)\right)=R E_{*} .
$$


The equation (56) defines two-dimensional surface in $\mathbb{R}^{3}$ of the pillow shape:

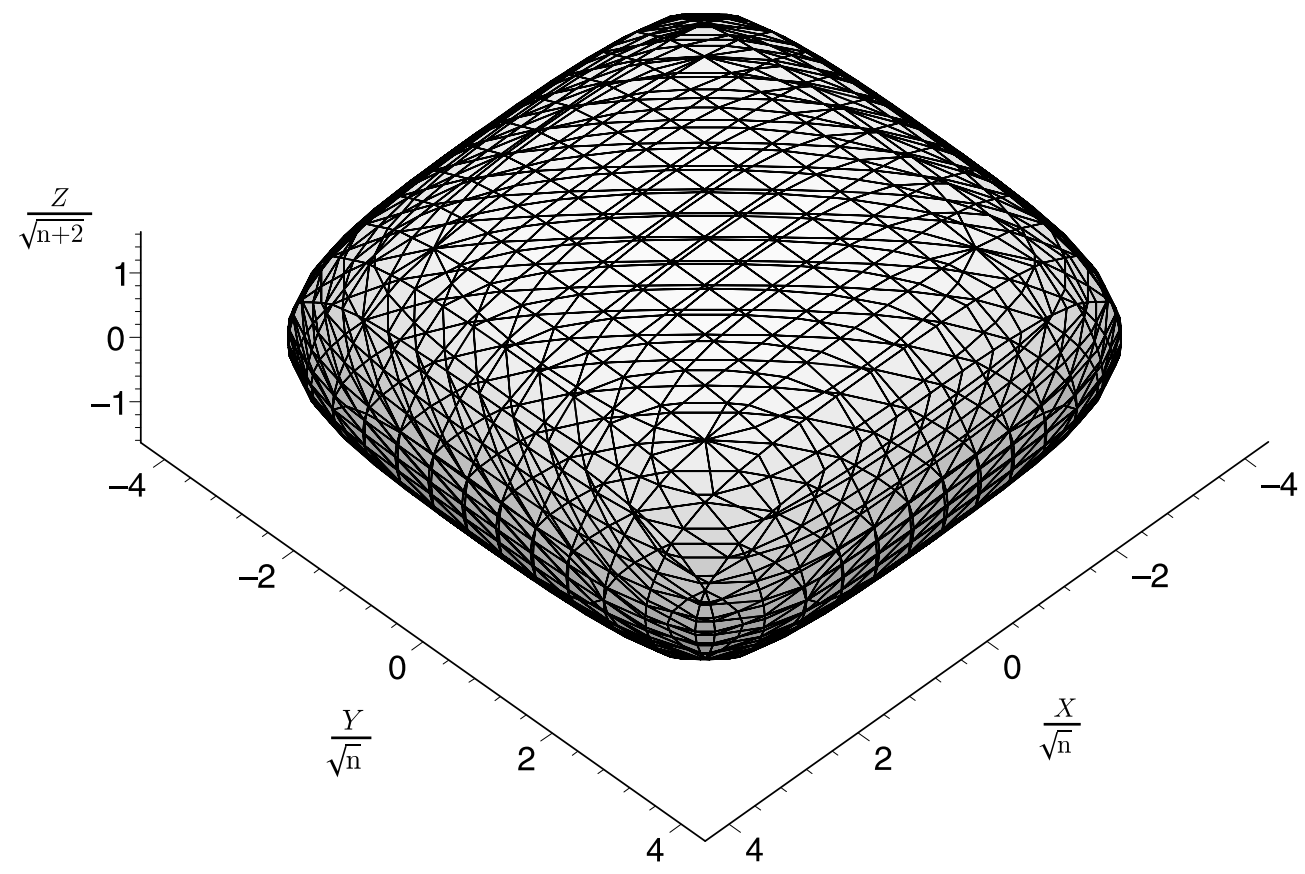

The action involves special $B$-field term,

$$
\mathscr{A}=\frac{1}{4 \pi} \int_{|z|<R}\left[\partial_{\mu} \mathbf{X} \partial_{\mu} \mathbf{X}-i \varepsilon^{\mu v} \mathbf{B}(X)\left(\partial_{\mu} \mathbf{X} \wedge \partial_{v} \mathbf{X}\right)\right] d^{2} x
$$

such that ${ }^{7}$

$$
\begin{aligned}
\left.B_{X}\right|_{\Sigma} & =-i \frac{a\left((1-v)^{2}-b^{2}\right)}{\sqrt{1-v} D} \sinh \frac{X}{\sqrt{v n}} \\
\left.B_{Y}\right|_{\Sigma} & =i \frac{b\left(v^{2}-a^{2}\right)}{\sqrt{v} D} \sinh \frac{Y}{\sqrt{(1-v) n}} \\
\left.B_{Z}\right|_{\Sigma} & =i \frac{b^{2} v^{2}-a^{2}\left((1-v)^{2}\right.}{\sqrt{v(1-v)} D} \sinh \frac{Z}{\sqrt{n}} .
\end{aligned}
$$

where

$$
a=\frac{\sqrt{\rho_{1} \rho_{2}}}{\left(1+\rho_{1}\right)\left(1+\rho_{2}\right)}, \quad b=\sqrt{\frac{1}{\left(1+\rho_{1}\right)\left(1+\rho_{2}\right)}},
$$

and

$$
D=a\left((1-v)\left(a^{2}-1\right)+(1+v) b^{2}\right) \cosh \frac{X}{\sqrt{v n}}+
$$

${ }^{7}$ Extended away from $\Sigma$, the $B$-field (59) is solenoidal, $\nabla \mathbf{B}=0$, so that the $B$-field term in (58) reduces to the boundary term

$$
-\frac{i}{2 \pi} \int_{0}^{2 \pi R} d y \mathbf{A}\left(X_{B}\right) \mathbf{e}_{j} \partial_{y} \eta^{j},
$$

where locally $\mathbf{B}=\nabla \times \mathbf{A}$, and $\eta^{j}$ are local coordinted on $\Sigma$. 


$$
b\left(v\left(1-b^{2}\right)+(2-v) a^{2}\right) \cosh \frac{Y}{\sqrt{(1-v) n}} .
$$

The pillow brane model is integrable. The corresponding differential equation has the form [5]

$$
\left[-\frac{d^{2}}{d w^{2}}+\frac{A^{2} e^{w}+C^{2}}{1+e^{w}}+\frac{B^{2} e^{w}}{\left(1+e^{w}\right)^{2}}+\left(E_{*} R\right)^{2} e^{-n v w}\left(1+e^{w}\right)^{n}\right] \Psi(w)=0,
$$

where the coefficients are expressed through the zero-mode momentum $\mathbf{P}=\left(P_{X}, P_{Y}, P_{Z}\right)$,

$$
C^{2}=-\frac{n v}{4} P_{X}^{2}, \quad A^{2}=-\frac{n(1-v)}{4} P_{Y}^{2}, \quad B^{2}=\frac{1}{4}-\frac{n+2}{4} P_{Z}^{2} .
$$

Conclusion: Recent advances of integrable field theories with boundary reveal remarkable relations between integrable boundary states, Baxter's families of commuting operators and local IM, and ordinary differential equations. These relations provide powerful tool for analysis of interesting models of boundary interactions, including the models with direct applications in Condensed Matter theory. At the same time, the deeper origin of these relations remains to be uncovered.

\section{References}

[1] V.Bazhanov, S.Lukyanov, A.Zamolodchikov, Integrable Structure of Conformal Field Theory I: Quantum KdV Theory, and Thermodynamic Bethe Ansatz, Commun.Math.Phys.177: 381-398 (1996), hep-th/9412229; Integrable Structure of Conformal Field Theory II: Q-Operator and DDV Equation, Commun.Math.Phys.190: 247-278 (1997), hep-th/9604044; Integrable Structure of Conformal Field Theory III: The Yang-Baxter Relation, Commun.Math.Phys.200: 297-324 (1999), hep-th/9805008.

[2] S.Lukyanov, A.Zamolodchikov, Integrable Circular Brane Model and Coulomb Charging at Large Londuction, J.Stat.Mech.0405: P003 (2004), hep-th/0306188

[3] S.Lukyanov, E.Vitchev, A.Zamolodchikov, Integrable model of boundary interaction: The Paperclip, Nucl.Phys.B683: 423-454 (2004), hep-th/0312168

[4] S.Lukyanov, A.Tsvelik, A.Zamolodchikov, Paperclip at $\theta=p i$, Nucl.Phys.B719: 103-120 (2005), hep-th/0501155

[5] S.Lukyanov, A.Zamolodchikov, In preparation.

[6] P.Dorey, R.Tateo, Anharmonic Oscillators, the Thermodynamic Bethe Ansatz, and Nonlinear Integral Equations, J.Phys.A32: L419-L425, (1999), hep-th/9812211; On the Relation Between Stokes Multipliers and the T - Q Systems of Conformal Field Theory, Nucl.Phys.B563: 573-602 (1999), hep-th/9906219

[7] V.Bazhanov, S.Lukyanov, A.Zamolodchikov, Spectral Determinants for Schrodinger Equation and $Q$ Operators of Conformal Field Theory, J.Statist.Phys.102:567-576,2001. e-Print: hep-th/9812247; Higher level eigenvalues of $Q$ operators and Schroedinger equation, Adv.Theor.Math.Phys.7: 711-725 (2004) hep-th/0307108

[8] S.Lukyanov, Notes on Parafermionic QFT's with Boundary Interaction, RUNHETC-06-01, Jun 2006, hep-th/0606155

[9] S.L. Lukyanov, P. Werner, Resistively Shunted Josephson Junctions: QFT Predictions Versus MC Results, RUNHETC-2007-03, Mar 2007, e-Print: cond-mat/0703712 